\title{
Progrès et inspiration dans le quotidien transgressif de Magritte
}

\section{Marcella Biserni}

\section{Q OpenEdition}

\section{Journals}

\section{Édition électronique}

URL : http://journals.openedition.org/rief/232

DOI : 10.4000/rief.232

ISSN : 2240-7456

\section{Éditeur}

Seminario di filologia francese

\section{Référence électronique}

Marcella Biserni, « Progrès et inspiration dans le quotidien transgressif de Magritte », Revue italienne d'études françaises [En ligne], 3 | 2013, mis en ligne le 15 décembre 2013, consulté le 19 avril 2019. URL : http://journals.openedition.org/rief/232 ; DOI : 10.4000/rief.232

Ce document a été généré automatiquement le 19 avril 2019.

\section{(c) (i) (9)}

Les contenus de la RIEF sont mis à disposition selon les termes de la Licence Creative Commons Attribution - Pas d'Utilisation Commerciale - Pas de Modification 4.0 International. 


\title{
Progrès et inspiration dans le quotidien transgressif de Magritte
}

\author{
Marcella Biserni
}

1 Les surréalistes affichent, d'une part, un certain refus des nouvelles technologies (par exemple le cinéma parlant), et, de l'autre, une attraction pour les différentes possibilités de reproduire les images, que ces moyens leur offraient. Pour expliquer cette ambivalence et en chercher la raison, je choisirai Man Ray et le mettrai en relation avec les définitions d'« expérience » et d'« expérimentation », que John Dewey exploite dans son Art as experience (1934). Forte de ces analyses, je m'intéresserai enfin au travail expérimental de René Magritte et au point limite de transgression dans le work in progress de sa création picturale ou cinématographique.

\section{L'expérimentation pragmatique dans l'« expérience » surréaliste}

2 La poétique de l'automatisme appliquée à l'art figuratif pousse les surréalistes à découvrir de nouvelles méthodes de création ${ }^{1}$ et à expérimenter d'autres procédés artistiques, outre la peinture. L'inquiétude, c'est-à-dire la mise en question de ce monde, et le refus de chercher hors de lui le salut restent au cœur de l'aventure surréaliste, autant que la recherche d'une vision renouvelée de l'ordre du monde permettant d'en dénoncer l'absurdité et de redonner sens à ce qui est reçu comme contingent, notre réalité la plus quotidienne. C'est ainsi, par exemple, que Walter Benjamin interprète la fétichisation surréaliste des objets démodés dans son article « Le Surréalisme. Le dernier instantané de l'intelligentsia européenne $»^{2}$ (1929) ; à ses yeux, l'investissement surréaliste du désuet et du débris crée un véritable «choc temporel » qui vise à briser l'illusion du progrès illusion conçue dans une dimension politique comme réaction à la civilisation bourgeoise et à son idéologie. Le choc perceptif ou mental, en détruisant l'habitus, doit ouvrir les portes à un remodelage anthropologique favorable à la vraie révolution. C'est à partir de ces mêmes conceptions militaro-stratégiques de l'art que le groupe français organise son 
offensive dans le champ culturel $^{3}$. La contradiction surgit, au moins apparemment, lorsqu'on souligne le fait que les membres du mouvement proposent d'entrée de jeu une solution qui garantit à l'homme une liberté réalisable, en se basant sur la recherche expérimentale et scientifique et en s'appuyant sur la philosophie et sur la psychologie.

Les surréalistes semblent suivre la pensée de Dewey, selon laquelle "l'un des traits distinctifs de l'artiste, c'est d'être né expérimentateur $»^{4}$. En effet, l'expérimentation permet au Surréalisme d'éviter le cliché et de s'éloigner d'un art académique. La nécessité de trouver de nouvelles formes d'expression expliquerait donc la discordance entre la théorie des surréalistes (déclarations écrites ou orales), contraires au progrès et surtout à l'industrialisation de l'art, et leur tendance à transgresser les moyens de création, aussi bien figuratifs que littéraires. Dans Art as experience John Dewey précise, en les appliquant à l'art, les principes du pragmatisme, qui procèdent d'une inspiration empirique et visent à des procédés d'observation et de contrôle expérimental des hypothèses. Expérience et expérimentation, pour le philosophe américain, constituent les noyaux de la méthode scientifique et doivent aussi être appliquées au domaine esthétique, l'hypothèse étant un processus d'invention libre. André Breton et les siens symbolisent cette typologie d'artistes ou d'écrivains qui ne sous-estiment pas le caractère relatif de la technique à l'égard des instruments ; au contraire, tous les militants du groupe s'en servent pour qu'il $\mathrm{y}$ ait renouvellement et dépassement dans l'urgence, affirme encore Dewey, de «trouver de nouveaux contenus d'expérience en quête d'expression et donc impliquant de nouvelles formes et techniques d'expression $»^{5}$.

\section{Man Ray un artisan de l'expérimentation}

Cette discordance entre expérimentation transgressive et attachement à l'« aura » et au talent de l'artiste est très présente dans les affirmations et les créations de Man Ray, qui travailla avec plusieurs machines-support: de l'appareil photographique à la caméra cinématographique. Le parcours de Man Ray se développe autour de l'expérience « expérimentale» des avant-gardes, qui nie le concept traditionnel d'expérimentation. Avec son œuvre de 1916, Self-Portrait, Man Ray avait abattu son premier atout : l'humour iconoclaste. Mais cette volonté de démythification et de désacralisation de l'art, qui l'a entrainé à utiliser des procédés " non artistiques ", ne suffit pas à justifier sa tendance à l'« expérimentation ».

5 En anglais le terme (to) experiment, traduit comme verbe ou comme substantif, désigne à la fois un événement accompli et un processus; elle enveloppe simultanément l'instant immédiat de la création, ou bien le moment de l'inspiration, et le produit final ; ce que John Dewey appelle l'« expérience aboutie ». Si on se réfère aux déclarations de Man Ray on peut trouver un trait d'union avec le philosophe américain car d'un point de vue esthétique, pour l'artiste surréaliste l'important n'est pas ce qu'un objet est, mais la façon dont il fonctionne dans l'expérience dynamique et le résultat de dépaysement qu'il provoque. Ces peintures à l'aérographe: The Rope Dancer (1916), La Volière, Admiration of the Orchestrelle for the Cinematograph, Seguidilla (1919), indifféremment abstraites ou figuratives, créent des espaces aériens, où la gouache pulvérisée rappelle certains effets photographiques. La réalité en est comme gommée, au bénéfice d'un pur acte cérébral. L'érotisme s'accentue dans $\mathrm{Mr}$ and Mrs Woodman qu'il réalise entre 1927 et 1945 en photographiant, sur fond blanc, des pantins de bois s'accouplant dans toutes les positions. Ces assemblages sont un clin d'œil à l'industrialisation qui fait directement écho aux 
peintures mécanomorphes qu'il a pu réaliser précédemment avec la complicité de Marcel Duchamp et parallèlement à Francis Picabia, pour évoquer la condition de l'homme moderne, changé en machine par la quête du progrès technique.

D'une part la recherche à travers l'expérience expérimentale; de l'autre, une critique radicale du progrès dont témoignent les déclarations de son autobiographie, Self-Portrait (1963), sur le film Retour à la raison (1923).

I had no idea what this would give on the screen. Also, I knew nothing about film mounting with cement so I simply glued the strips together, adding the few shots first made with my camera to prolong the projection. The whole would not last more than about three minutes. Anyhow, I thought, it would be over before an audience could react; there would be other numbers on the program to try the spectators' patience, the principal aim of the Dadaists ${ }^{6}$.

Man Ray marche dans les pas de Marcel Duchamp sur les traces de l'« objet trouvé » ou de la «trouvaille », sans se préoccuper de la reproductibilité de l'œuvre. Dans ce film, il fait preuve d'une exceptionnelle habileté en complète opposition avec les expérimentations dictées par la machine-support, mais d'un autre côté, encore une fois, cette œuvre est liée à l'idée de progrès et traduit une attraction vers l'expérimentation du moyen technique ${ }^{7}$. En 1936 ses déclarations pour expliquer les techniques employées dans la réalisation d' Emak Bakia (1926) ne laissent pas d'espace à la discussion: « And I was thrilled, more with the idea of doing what I pleased than with any technical and optical effects I planned to introduce $»^{8}$. Le sujet « expérimentation » semble pour lui clair et irréversible: «I never showed my experiments - what I offered to the public was final, the result of a way of thinking as well as seeing ${ }^{9}$; ses œuvres, en résumant sa pensée, sont des expériences abouties. Ce qu'on montre au spectateur n'est pas la technique, mais l'improvisation transformée et mise au point par des expérimentations. Le résultat du travail obtenu est la transposition parfaitement réussie de ce que l'artiste avait à l'esprit et ressentait au moment de l'illumination, de son inspiration ${ }^{10}$. L'utilisation des instruments est une espèce d'épreuve toujours perfectible, tandis que l'expérience implique le concept d'une « réalisation » aboutie à offrir au public ${ }^{11}$. C'est le cas de Retour à la raison et aussi d'Emak Bakia, où Man Ray manipule le matériel avec violence (la pellicule), comme il l'avait fait en photographie avec les rayographes, en arborant une sorte de mépris à l'égard de la machine, voire de l'art. Ce type d'expérimentations cherche à provoquer le dépaysement chez le spectateur, à travers la transgression des moyens de création habituels, guidés par le hasard et l'humour en dépit des supports mécaniques. Ses œuvres-collages sont donc des travaux de haute manufacture; des bricolages créés non par goût du progrès mais par désir de transgresser les règles et de donner par là un sens nouveau à la réalité. C'est la sensation provoquée chez le spectateur qui est au centre de la création et non pas la technique employée pour y parvenir. Dans cette perspective, on peut concevoir la désillusion de Breton et de ses compagnons au moment de l'arrivée du cinéma parlant ; ce technicisme trop étalé et trop conditionné par la recherche esthétique, réduit le cinéma moderne à un simple formalisme pauvre d'idées et d'objectifs. Pour ces raisons, les expérimentations de Man Ray ne peuvent être considérées elles non plus uniquement comme des œuvres accomplies sans tenir compte de l'instrument utilisé.

\section{Magritte et la transgression conservatrice}


de son œuvre pluridisciplinaire. Tout au long de sa carrière, outre qu'à la peinture, son art touche à l'illustration publicitaire et littéraire, à la photographie et au cinéma et dans tous ces domaines Magritte résout (peut-être sans le vouloir) la contradiction entre théorie et pratique, en agressant le mur du quotidien, en le transgressant. En effet, ses créations ne nient pas sa tension vers l'expérimentation en peinture, marquée par le trompe-l'œil et les effets spéciaux (déplacement de l'ordre naturel des objets, changement des proportions, révolution des matériaux, inversion de perspective) qui multiplient le merveilleux quotidien. La plasticité de l'image tourne autour de deux piliers portant la plateforme des métamorphoses magrittiennes: l'humour et une certaine dose d'érotisme. Les figures se plient complètement au désir intérieur des choses jusqu'à perdre tout système de référence, en rentrant dans un nouvel univers de pure transgression et de bouleversement. La visée est bien révolutionnaire et la transgression passe par le fil rouge de l'humour, à maintes reprises érotique, comme déjà chez Man Ray. Il semblerait que le penchant qui le portait à manipuler ses images récurrentes avec les différentes techniques - en phase de développement à son époque (photographie et cinéma) - ait évolué à partir de la même structure philosophique et idéologique que celle qui caractérisait sa peinture.

9 Ses déclarations sur les nouvelles technologies contrastent en revanche avec sa production artistique interdisciplinaire. Les écrits de Magritte donnent une formulation théorique très claire de sa production picturale, qui soutient la représentation figurative de façon conceptuelle de la même manière que la traduction de la pensée en images dans ses œuvres. Ses trompe-l'œil changent le jour en nuit (L'Empire des lumières, 1947) et rendent vraisemblable le paysage au-delà du cadre/fenêtre (La Condition humaine, 1933) jusqu'à transformer l'artiste en magicien (Le Sorcier, 1951 et Le Domaine d'Arnheim, 1962). Comme Magritte lui-même le déclare : « un paysage nocturne sous un ciel ensoleillé et d'autre part une montagne en forme d'oiseau, démontrent que la peinture - telle que je la conçois - n'est pas orientée par des recherches formelles, mais concerne la pensée, exclusivement par son manque de solution de continuité avec le monde et son mystère $»^{12}$

10 Pour ce qui concerne les supports, tout particulièrement en référence au cinéma, il affirme : «Que chacun d'entre nous garde les moyens techniques de sa génération, sinon la parole sera toujours au dernier venu et rien, jamais, ne sera dit $»^{13}$. À vrai dire, la contradiction qui surgit entre ces déclarations et sa pratique artistique révolutionnaire n'est qu'apparente ; car, encore une fois, comme dans le cas de Man Ray, il s'agit d'agir et d'inventer des moyens d'action toujours renouvelés dans une démarche dialectique, où l'expérimentateur ne s'identifie pas à son outil. En 1941, Paul Nougé reconnaît cette caractéristique au surréalisme ${ }^{14}:$ "J'ai déjà fait remarquer cependant combien ils (les surréalistes parisiens) ont toujours été fidèles, en fait, aux grandes disciplines expérimentales, et cela, dans des domaines où n'avait régné jusqu'ici que le vague et l'arbitraire $\aleph^{15}$. Mais si les futuristes dès le début construisent leur révolution sur la base des machines et du progrès, en dépit de leur maîtrise des nouvelles techniques, les surréalistes au contraire hésitent à y avoir recours, du moins au niveau théorique. Autrement dit, on peut constater qu'ils n'acceptent pas ouvertement le défi et le risque des techniques modernes, encore trop peu connues, où la compétence artistique de l'expérimentateur pouvait être jugée médiocre et le résultat une mauvaise tentative de création transgressive. Magritte lui-même garde ses distances avec la caméra cinématographique, en déclarant «Je ne fais pas du ciné, pas de cinéma, je fais du 
cinématographe $»^{16}$. Cette affirmation prend la forme d'un cryptogramme construit sur la parole " cinéma ", de manière à provoquer un rébus à la façon typiquement magrittienne, une sorte d'idéogramme graphique. Il s'agit, en effet, d'un jeu de mot qui dans un crescendo des lettres ajoute des morceaux phonétiques et figuratifs à la " graphie » écrite, jusqu'à créer l'univers "ciné-ma-(to)-graphique ». L'artiste belge semblerait vouloir mettre l'accent sur le fait que le septième art est, avant tout autre chose, une pratique d'écriture des images; juxtaposées les unes après les autres, comme déjà ces objetsfétiche dans ses tableaux. Il soulignerait, donc, l'aspect technique de la construction des images en mouvement, en la renvoyant à sa caractéristique de cinéma primitif, où l'élément expérimentation se limitait à l'entrelacement entre les mots des génériques ou des légendes et les figures projetés dans l'écran.

On est quand même face à une transgression quand on regarde ses films, censurée et dans un certain sens refusée, mais qui devient puissante et bouleversante au moment de la concrétisation de l'inspiration artistique. Magritte est attiré, comme tous les surréalistes, français et belges, par les moyens artistiques, mais d'un autre côté, il semblerait lui aussi se retenir vis-à-vis de leur utilisation, à cause d'un manque de maitrise dans ces nouveaux domaines (photographie et cinéma). Ce n'est pas un hasard, par exemple, si les films de Magritte sont muets ${ }^{17}$. Dans son cinéma, comme dans sa peinture, l'image est au centre de la représentation avec comme seul but de toucher le spectateur et de le surprendre à partir de ce qui est déjà là, notre réalité la plus quotidienne. La vision des choses et de la banalité dans leur mouvement perpétuel prend le pouvoir sur l'expression communicative ou narrative, sur les traces de sa production picturale, sans trop toucher aux virtuosités du technicisme. Le seul effet que le peintre bruxellois tient en considération paraît être celui du montage. L'assemblage manuel de la pellicule, en effet, comme l'affirme aussi Walter Benjamin dans son L'Art à l'époque de sa reproductibilité technique ${ }^{18}$, se rapproche beaucoup du collage ; moyen de création que Magritte appréciait et dont il avait loué l'usage chez Max Ernst.

12 En illustrant «Répétitions » de Paul Éluard, Max Ernst a démontré magnifiquement par l'effet bouleversant de collages obtenus au moyen de vieilles gravures de magazine que l'on pouvait aisément se passer de tout ce qui donne son prestige à la peinture traditionnelle. Les ciseaux, de la colle, des images et du génie ont remplacé, en effet, les pinceaux, les couleurs, le modèle, le style, la sensibilité et le souffle sacré des artistes ${ }^{19}$.

Sous l'égide du collage, le montage devient le fil rouge entre intertexualité et interdisciplinarité : parcours presque obligé, selon Magritte, pour montrer le croisement des analogies dans les choses et leur «sympathie» (Foucault) interne ${ }^{20}$. Le cinéma procède de la même manière que la peinture : il s'agit d'un art de la citation qui s'inspire de la réalité, ou ne fait que la suggérer, en ouvrant les portes à un nouvel espace interprétatif où l'aura perdue peut être retrouvée. Par le biais de l'humour Magritte colle les images de façon paradoxale et fantasmagorique, en ajoutant dans le support pellicule la possibilité de les faire bouger. Pierre Sterckx le premier, et le seul à notre connaissance, a souligné comment et combien le peintre belge cherche dans le cinéma et en photographie à dominer le temps. Le critique en voit la suggestion la plus directe dans Le Dessert des Antilles (1957) : ici une banane est reconstruite à l'envers et «Le temps est rebobiné. Est vaincu $»^{21}$. Le cinéma pour René Magritte, et peut-être pour le surréalisme tout entier, n'est donc qu'une machine temporelle qui renverse chaque image vue et vécue à la manière d'une métalepse. La cause et l'effet se retournent et, poursuit Pierre Sterckx en se référant au film de Magritte : «Le cours du temps, désorienté, désarticule 
aussitôt l'organicité du corps [...] $»^{22}$; l'image se transforme en une spirale capable de parcourir de façon entropique ce que la réalité impose : la vie et ensuite la mort. Les images picturales de Magritte, même les plus sophistiquées, froides et imperturbables, vivent de mouvement et de ce rythme de la nature à imiter, que John Dewey avait exalté lui aussi, et elles trouvent leur concrétisation dans la pellicule. La caméra, comme la chambre obscure, joue le rôle du masque qui dévoile ce qui se trouve au-delà de l'apparence et du visible ; elle est pour l'artiste belge un moyen de travestissement du réel et de son renversement, qui révèle le punctum, dirait Barthes, dans l'image de l'objet la plus banale, dans ce cas une banane.

Pourtant, nonobstant sa pratique des machines, Magritte détourne le regard du sujet expérimentation, car avant d'arriver au moyen, à l'action et à l'expérience de la création, il importe de cueillir l'instant magique. Georgette Magritte se rappelle que son mari "parlait de la "pensée inspirée", c'est-à-dire de l'inspiration, une inspiration qui n'était

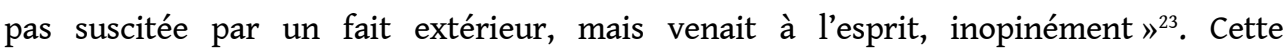
illumination devait déboucher sur l'action performative et arriver jusqu'au sein de la complexité universelle. En ce sens, le parallèle avec Dewey est fort intéressant surtout si l'on pense à l'idée intrinsèque dans son Art as experience, selon laquelle l'artiste devrait remettre en question les règles, en les transgressant. Le philosophe américain invite à chercher une réponse qui modifie les rapports de la vie quotidienne avec le reste de l'univers: "Car les œuvres nouvelles, dans la mesure où elles sont bien nouvelles, ne correspondent pas à des cases déjà toutes prêtes. [...] Les règles qui président aux classifications ajoutent un handicap de plus $»^{24}$. Le philosophe américain insiste ensuite sur l'importance décisive du moyen de la création et de ses matériaux et souligne le fait que : «[...] les limites exactes de l'efficace d'un médium ne peuvent être déterminés par aucune règle a priori et que tout grand innovateur en art brise certaines barrières que l'on supposait auparavant infranchissable $\aleph^{25}$. Ces mots nous renvoient directement à l'idée que le geste de la création, entendu comme expérience in itinere et expérimentale, fait la part belle au regard, en tant qu'électeur de l'objet d'art ${ }^{26}$, dans la lignée de Man Ray et de Magritte.

Le surréalisme, Magritte compris, ne peut pas se passer d'expérimenter les nouveaux moyens de création, photographie et cinéma en particulier, car ils représentent un enjeu, ou plutôt la possibilité de retrouver l'« aura » perdue du fait de la réification industrielle de l'art. Mais si Man Ray, comme Marcel Duchamp et Francis Picabia, renonce de plus en plus à démontrer l'originalité et l'unicité de l'artiste à travers la peinture, inaugurant une nouvelle époque où l'objet n'a plus besoin d'être peint, Magritte, au contraire, reste attaché à son métier de peintre. Il cherche par là, par la peinture en premier lieu, à nous montrer une réalité tellement vraisemblable qu'elle devient artificielle. Son hyperréalisme se transforme en exagération du désir d'inconnu et de dévoration du quotidien. Dans ses photographies ou dans ses films, comme dans ses tableaux, il n'existe plus une suprématie de l'objet réel et l'existence se trouve désormais en contact avec un tourbillon d' " expériences » et de " perceptions ", termes chers à Dewey, où la machine produit un équivalent visuel de la parole magique et de l'image merveilleuse. 


\section{La tentation du mouvement selon Magritte jusqu'à nos jours}

16 L'expérimentation est donc glorifiée par les surréalistes uniquement en tant que moyen pour aboutir au-delà de l'illusion du réel. Les machines sont de simples instruments au service de l'inspiration, mais, malgré tout, devant ces prises de position les surréalistes, et Magritte tout particulièrement à notre avis, ne résistent pas à la tentation de pouvoir animer les images, de les superposer et de les transformer. Comment ne pas prendre en considération la possibilité de changer l'irréversible et de transgresser les règles de la nature à travers les machines? Le cinéma et la photographie semblent être surréalistes dans leur essence ; ces techniques pouvaient rendre réel ce fantastique et ce merveilleux si près du quotidien et en même temps invisibles à nos yeux.

17 Magritte en peinture, par les couleurs et la toile, compose de nouvelles combinaisons, mêlant le réel et le rêve exactement comme le cinéma avait commencé à le faire depuis un demi-siècle. La réalité qu'il met en scène devient aujourd'hui la seule protagoniste de l'art du mouvement dans un effet d'animation que le peintre avait pu seulement imaginer. Dans le cinéma contemporain, la technique des effets spéciaux du morphing se présente comme le développement naturel des méthodes picturales magrittiennes de nos jours $^{27}$. Il n'y a pas de filiation directe de l'un à l'autre, mais il est possible de renvoyer les deux à la même matrice transformiste et au même désir de transgression. L'important, comme dirait Man Ray, est le résultat et le fait qu'avec le morphing on peut rendre possible l'impossible à travers une métamorphose progressive de l'objet, précisément comme certains tableaux de Magritte le suggèrent. On ne peut pas savoir si Magritte aurait utilisé cette technique informatisée, mais il est bien certain qu'elle s'est emparée de son inspiration.

18 Et on est alors tenté de se demander si les manipulations de ses œuvres réalisées aujourd'hui à l'ordinateur ennuieraient Magritte, comme quelques-uns des films qu'il avait vus, ou si elles le surprendraient. Dans le court-métrage de James Kelly ${ }^{28}$ tout ce que Magritte voulait résumer dans la peinture, à travers la photographie et dans le cinéma trouve maintenant une synthèse. Les objets-fétiches, décrits dans toute sa production, prennent vie et donnent un sens à la recherche de ce mouvement tant rêvé. Les hommes au chapeau melon de Golconde (1953) ne restent plus en suspension dans le ciel, mais ils tournoient et se dissipent dès qu'ils touchent le sol. Les matériaux se fondent et les objets se transforment si rapidement qu'ils se confondent les uns avec les autres ${ }^{29}$. La statue victime étendue sur le canapé de L'Assassin menacé (1926) - ressuscite au son du phonographe et l'espion malfaiteur masqué derrière la porte ne reste pas caché, mais plaque la femme-statue contre un mur et avec une rose blanche guérit sa blessure. Désormais la sculpture peut s'incarner en Vénus colorée comme dans La Magie noire (1935).

19 La réalité tout entière devient interchangeable, chaque chose peut changer de forme et de matériau, et si André Breton et ses amis, sous l'égide de Lautréamont, utilisaient les mots pour donner vie à des métamorphoses ou à des métaphores, le peintre se trouve juste au centre, à mi-chemin, entre cette technique et celles qu'on connait aujourd'hui. L'effet de ses «transgressions" digitales, qui ajoutent le mouvement aux métamorphoses de l'image peinte, bouleverse le spectateur. Cette sensation d'inquiétude et de dépaysement que les surréalistes recherchaient, en entrant au hasard dans une salle 
cinématographique ou derrière les masques et les visages maquillés des acteurs, ressemble probablement à celle que le spectateur d'aujourd'hui ressent devant une métamorphose tridimensionnelle numérique. On peut penser, par exemple, pour en rester à un tableau de Magritte, aux transformations des personnages aux lunettes noires, tous pareils et anonymes, dans The Matrix des frères Lana e Andy Wachowsky (1999), qui ressemblent beaucoup aux hommes au chapeau melon de Golconde. Dans les deux versions, cinématographique et picturale, le spectateur est plongé dans un monde habité par des automates, qui se reproduisent à l'infini en plongeant du ciel et saisissant les formes d'autres corps. Le prêt que les deux réalisateurs semblent avoir tiré de la pensée magrittienne n'est pas seulement figuratif ; à la base de cette cosmographie transgressive, en effet, on retrouve la même vision métaphorique représentant la banalité du monde, sur les traces de la banalisation du quotidien magrittienne. Le résultat obtenu dans l'une et dans l'autre création artistique génère chez le spectateur un refrain, ou plutôt une arrière-pensée, très similaire à une autre toile de l'artiste, La Trahison des images (1927). Il s'agit d'une sorte de négation dans la négation, qui met en marche une reformulation de la réalité même et qui fait exclamer dans une imprévisible inspiration révélatrice : «Ceci n'est pas la réalité ! $»^{30}$.

\section{NOTES}

1. Certaines de ces méthodes n'étaient pas originales, le collage par exemple fut expérimenté pendant la période dadaïste, comme la peinture et la sculpture d'objets, mais le surréalisme en augmenta encore plus la portée avec l'implication d'autres sens outre la vue.

2. W. Benjamin, «Le Surréalisme. Le dernier instantané de l'intelligentsia européenne », dans Euvres II, Paris, Gallimard, « Folio Essais », 2000, p. 130.

3. Ce rejet du développement technologique paraît être une caractéristique propre aux avantgardes françaises, alors que plusieurs autres groupes européens défendent une vision apologétique de la modernité technique (futurisme, constructivisme, etc.). Cf. A. Trudel, «Des surréalistes aux situationnistes. Sur le passage entre le rêve et l'ivresse ", COnTEXTES, n. 6, septembre 2009, <http://contextes.revues.org/index4421.html>, mis en ligne le 25 septembre 2009, consulté le 11 juin 2013.

4. J. Dewey, L'art comme expérience, Paris, Gallimard, « Folio essais», 2010, p. 45 (éd. or. : Art as experience, New-York, Minton, Balch and Co., 1934).

5. Ibid., p. 244.

6. M. Ray, Self-Portrait, London, Bloomsbury, 1988, p. 212 (éd. or. : Boston, Little-Brown, 1963).

7. Cf. G. Angeli, Macchine meravigliose. Surrealismo e tecnologia, Firenze, Le Lettere, 2009.

8. M. Ray, op. cit., p. 219.

9. Ibidem.

10. G. Angeli, op. cit., p. 123.

11. Ibid., p. 122, 123.

12. R. Magritte, Écrits complets, édité par André Blavier, Paris, Flammarion, 1976, p. 552.

13. M. Rapin, Aporismes, Viry-Chatillom, S.E.D.I.E.P., 1970, p. 26, 27.

14. G. Michel, Paul Nougé. La poésie au cœur de la révolution, Bruxelles, Peter Lang, 2011, p. 86.

15. Ibid., p. 81. 
16. L. Scutenaire (textes et titres par), La Fidélité des images, René Magritte. Le cinématographe et la photographie, Musées Royaux des Beaux-Arts de Belgique, 14 mai -11 juillet 1976, Bruxelles, Éditions Musées Royaux des Beaux-Arts de Belgique, 1976, p. 70.

17. Ces pellicules, tournées avec la collaboration de son entourage entre 1956-1967, furent coupées en 1965 par Robert Rimbaerd (spécialiste du montage et employé à la RTBF) et Jean Raine (artiste près de Scutenaire), mais le montage ne fut jamais terminé. Pour cette raison, il ne reste que des morceaux de ces films. Cf. B. Verschaffel et J. Cornelis, «Les films d'amateur de René Magritte ", dans Magritte, Catalogue du Centenaire, G. Ollenger-Zinque et F. Leen (dir.), Musée des Beaux Arts de Bruxelles, Bruxelles, Ludion-Flammarion, 1998, p. 286-288, p. 286. Signalons à ce propos notre ouvrage consacré aux films magrittiens, M. Biserni, Magritte e il cinema... chapeau !, Perugia, Morlacchi Editore, 2011.

18. Benjamin lui-même affirme : «Si la lithographie contenait virtuellement le journal illustré, la photographie contenait virtuellement le cinéma». W. Benjamin, L'Euvre d'art à l'époque de sa reproductibilité technique, in CEuvres III, traduit de l'allemand par M. de Gandillac, R. Rochlitz et P. Rusch, Paris, Gallimard, «Folio essais », 2000, p. 272 (dans la première version rédigée en 1935 cette citation se trouvent à la page p. 71). En 1936 Benjamin apporte d'importantes modifications au texte, en vue de sa publication et d'une rédaction en français. Une quatrième version, la dernière, date de 1939. Le « réveil » de la conscience collective, selon la conception de Benjamin, se fait sous forme de montage. Pour le philosophe allemand, cette technique, empruntée à la photographie et au cinéma, repose sur un enchaînement de citations, qui engendre le passage du rêve au réveil et par là à la mémoire.

19. R. Magritte, Écrits complets, op. cit., p. 104. Comme l'a remarqué Arturo Schwarz le ready-made, le plagiat, le collage tout comme le montage doivent beaucoup à l'influence de Ducasse. Il suffit de penser à la Fontaine ou au Porte-bouteille de Duchamp pour comprendre que l'assemblage n'est qu'une révision plastique de la formule ducassienne de la rencontre fortuite entre la machine à coudre et le parapluie. A. Schwarz, The complete works of Marcel Duchamp, Thames and Hudson, London 1969; New York, Delano Greenidge, 1977. Cf. L. Durand-Dessert, Lautréamont et les arts visuels (1970-1998), "Cahiers Lautréamont», Les lecteurs de Lautréamont, Actes du colloque international sur Lautréamont Montréal 5-7 octobre 1998, textes réunis par J.-J. Lefrère et M. Pierssens, Du Lérot éditeur, livraison XLVII et XLVIII, 2ème semestre 1998, p. 102.

20. Magritte témoigne son intérêt pour les théories foucaultiennes dans une lettre à Marcel Lecomte, datée avec des numéros, dans ce cas 6-4-2, sans donner la possibilité au lecteur de remonter à l'année exacte de la missive (pratique assez habituelle du peintre). Dans cette occasion, on assiste à un échange de réflexions avec l'ami poète sur la " pensée qui voit » et sur la question du visible, dans le spécifique l'artiste fait un raisonnement complexe sur le concept de représentation, que Foucault avait envisagé dans ouvrage de 1966: "Tu dis très bien que le possible de l'impossible s'accomplit! Et, en effet, la description visible (une image peinte) d'une pensée invisible (constituée par des figures visibles unies dans un certain ordre) est de "l'impossible possible" ou, en d'autres termes de "l'invisible visible". P. S. Je lis un livre très intéressant: "Les Mots et les choses" de Foucault (Gallimard) ». Archives des Musées Royaux des Beaux-Arts de Bruxelles (MRBAB-AACB), $n$. d'inventaire 8.905. Les guillemets et les soulignés sont de l'auteur.

21. P. Sterckx, «Magritte (cinéaste) », dans G. Jungblut, P. Leboutte et D. Païni (édition établie par), Une Encyclopédie des cinémas de Belgique, Musée d'Art Moderne de la Ville de Paris, Paris, Éditions Yellow Now, 1990, p. 184-186, p. 185.

22. Ibid., p. 186.

23. R. Passeron, René Magritte, Paris, Filipacchi, 1970, p. 12.

24. J. Dewey, op. cit., p. 371.

25. Ibid., p. 372. 
26. Cf. M. Nachtergael, "Images, désir et sacrifice ", Hal. Sciences de l'Homme et de la Société [en ligne], 27 octobre 2012 [réf. du 29 octobre 2012], 1-17, <http://halshs.archives-ouvertes.fr>.

27. Les effets spéciaux furent expérimentés pour la première fois par Georges Méliès à la fin du XIX siècle avec les films à trucs (trick films). Depuis, ces expérimentations-maison ont traversé diverses étapes de transformation jusqu'à aboutir aux techniques modernes. Avant le morphing, l'image était transformée seulement de façon numérique ou par les effets créés en laboratoire ( trucages). Les tentatives qui transforment au début l'image synthétique totalement informatisée ne donnent que des résultats très médiocres, comme dans Tron de Steven Lisberger (1982) ou Terminus de Pierre-William Glenn (1987). L'image synthétique, comme le souligne son nom, propose une synthèse de tous les effets spéciaux traditionnels, en prolongeant leur impact. Jusqu'à l'arrivée de cette technique, l'usage de l'ordinateur se limitait à l'image numérique qui était le trait-d'union ou le passage à l'utilisation plus moderne des effets spéciaux informatisés. Avec le morphing, l'image synthétique a acquis la mutation à laquelle elle est indissolublement liée, devenant de nos jours une pratique courante du cinéma.

28. J. Kelly, This is not a film, 2003, copyright Charlie Herscovici, <http://vimeo.com/8529097>

29. Déjà Georges Méliès, à la fin du film Les 400 farces du diable (1908), faisait descendre du ciel un homme avec un parapluie.

30. Cf. S. Žižek, "The Matrix o i due volti della perversione. Riflessi sulla virtualità cinematografica », Deleuze e il cinema, M. Bertolini e T. Tuppini (a cura di), Milano, Mimesis, 2002.

\section{INDEX}

Mots-clés : surréalisme, Man Ray, Dewey (John), Magritte (René), aura de l'artiste, illusion du progrès, expérimentation transgressive, peinture, cinéma, photographie, reproductibilité de l'œuvre d'art, transgression conservatrice 